\title{
BMJ Open Inappropriate prescribing among older persons in primary care: protocol for systematic review and meta-analysis of observational studies
}

To cite: Lee CS, Liew TM. Inappropriate prescribing among older persons in primary care: protocol for systematic review and metaanalysis of observational studies. BMJ Open 2017;7: e015395. doi:10.1136/ bmjopen-2016-015395

- Prepublication history and additional material is available. To view please visit the journal (http://dx.doi.org/ 10.1136/bmjopen-2016015395).

TML and CSL contributed equally to this work.

Received 2 December 2016 Revised 13 January 2017 Accepted 2 February 2017

CrossMark

${ }^{1}$ SingHealth Polyclinics,

Singapore

${ }^{2}$ Saw Swee Hock School of

Public Health, National

University of Singapore,

Singapore

${ }^{3}$ Department of Geriatric

Psychiatry, Institute of Mental Health, Singapore

Correspondence to

Dr Tau Ming Liew;

tau_ming_liew@imh.com.sg

\section{ABSTRACT}

Introduction: Inappropriate prescribing has a significant impact on older persons in primary care.

Previous reviews on inappropriate prescribing included a heterogeneous range of populations and may not be generalisable to primary care. In this study we aim to conduct a comprehensive systematic review and metaanalysis of the prevalence, risk factors and adverse outcome associated with inappropriate prescribing, specifically among older persons in primary care.

Methods and analysis: We will search PubMed, Embase, CINAHL, Web of Science, Scopus, PsycINFO and references of other review articles for observational studies related to the keywords 'older persons', 'primary care' and 'inappropriate prescribing'. Two reviewers will independently select the eligible articles. For each included article, the two reviewers will independently extract the data and assess the risk of bias using the Newcastle-0ttawa Scale. If appropriate, meta-analyses will be performed to pool the data across all the studies. In the presence of heterogeneity, meta-regression and subgroup analyses will also be performed. The quality of the evidence will be assessed using the Grading of Recommendations Assessment, Development and Evaluation (GRADE) approach.

Ethics and dissemination: The results will be disseminated through conference presentations and peer-reviewed publications. They will provide consolidated evidence to support informed actions by policymakers to address inappropriate prescribing in primary care, thus reducing preventable and iatrogenic risk to older persons in primary care.

Trial registration number: CRD42016048874.

\section{INTRODUCTION \\ Rationale}

Inappropriate prescribing refers to the practice of prescribing medications with potentially greater risk than benefit. ${ }^{1}$ Holistic definitions of inappropriate prescribing have been suggested to encompass the assessment of prescriptions to older persons in the context of their multiple comorbidities,

\section{Strengths and limitations of this study}

- This systematic review and meta-analysis wil provide a comprehensive summary of the various aspects of inappropriate prescribing among older persons in primary care.

- The results will provide the highest level of evidence that can be generalised to primary care and will facilitate informed actions by policymakers.

- This protocol has been developed in accordance with the Preferred Reporting Items for Systematic Review and Meta-analysis Protocols (PRISMA-P) statement and has been registered with the Prospective Register of Systematic Reviews (PROSPERO).

- The overall quality of evidence will be assessed using the Grading of Recommendations Assessment, Development and Evaluation (GRADE) approach.

- This systematic review will be limited to studies in English language.

complex medication regimes, functional and cognitive status, treatment goals and life expectancy. ${ }^{2}$ However, such holistic definitions are generally more challenging to operationalise in routine practice. Simpler definitions of inappropriate prescribing have been used by studies in the literature, including explicit (criterion-based) tools such as the Beers and Screening Tool of Older Persons' potentially inappropriate Prescriptions (STOPP) criteria and implicit (judgement-based) tools such as the Medication Appropriateness Index. ${ }^{34}$

Inappropriate prescribing is a form of medical error ${ }^{5}$ which is common and preventable in primary care. Its prevalence among older persons in primary care ranges from $16 \%$ to $60 \% .^{6-13}$ Due to the changing pharmacokinetics and pharmacodynamics in older persons, inappropriate prescribing increases the risk of adverse effects in this population and leads to adverse drug reactions, morbidity and mortality. ${ }^{14} 15$ From the societal perspective, inappropriate prescribing 
results in higher healthcare cost due to increased hospitalisations, outpatient visits, emergency department visits and nursing home placements. ${ }^{1}$ With recent evidence in the British Medical Journal highlighting medical error as the third leading cause of death, ${ }^{16}$ there is an urgent need for action to address common and preventable areas of medical error in primary care such as inappropriate prescribing.

To facilitate informed actions by policymakers, the extant literature on inappropriate prescribing in primary care needs to be consolidated to provide a clearer understanding of the problem. However, to date, no systematic review or meta-analysis has comprehensively studied the prevalence, risk factors and adverse outcomes of inappropriate prescribing specific to the primary care setting. Although some of the previous reviews have included primary care populations, their results may not be generalisable to primary care settings due to the heterogeneous range of included populations, such as those from tertiary healthcare settings or nursing homes. ${ }^{1} 41718$

\section{Objectives}

In this study we intend to focus exclusively on the population of older persons in primary care. We seek to conduct a comprehensive systematic review and meta-analysis to evaluate the prevalence, risk factors and adverse outcomes associated with inappropriate prescribing among older persons in primary care. Specifically, we aim to address the following research questions:

1. What is the pooled prevalence of inappropriate prescribing among older persons in primary care?

2. What is commonly used as the working definition of inappropriate prescribing among older persons in primary care, and how do the various working definitions affect the prevalence?

3 . What is the pooled effect size of each risk factor associated with inappropriate prescribing among older persons in primary care?

4. What is the pooled effect size of each adverse outcome associated with inappropriate prescribing among older persons in primary care?

\section{METHODS AND ANALYSIS}

This protocol is developed in accordance with the Preferred Reporting Items for Systematic Review and Meta-analysis (PRISMA) statement. ${ }^{19}{ }^{20}$ It has been registered with the International Prospective Register of Systematic Reviews (PROSPERO) (registration number CRD42016048874).

\section{Eligibility criteria}

Participants and settings

We will include studies:

- which recruited participants from primary care settings;

- with $\geq 90 \%$ of the participants who are $\geq 60$ years old;
- with $<90 \%$ of the participants who are $\geq 60$ years old, but reported subgroup analyses on participants who are $\geq 60$ years old.

Studies will be excluded if the recruitment was not from primary care settings, such as those from tertiary hospitals or nursing homes.

\section{Exposure and outcome measures}

We will include studies which report on the prevalence, risk factors or adverse outcomes related to inappropriate prescribing. We will include all types of adverse outcomes that have been reported in the literature, including but not limited to those related to adverse drug reactions, emergency department visits, hospitalisation and mortality. We will exclude studies that:

- are not related to inappropriate prescribing;

- do not have a working definition of inappropriate prescribing;

- only focus on inappropriate prescribing related to specific groups of medications.

\section{Study designs}

We will only include observational studies such as crosssectional, case-control and cohort studies. We will exclude the following study designs: qualitative studies, intervention studies, reviews, meta-analyses, case reports, case series, ecological studies, conference proceedings, letters, comments and policy papers.

\section{Language and time frame}

We will only include studies which are reported in the English language. We do not impose any time restriction to the publication year of the studies.

\section{Information sources and search strategy}

We will search PubMed, Embase, CINAHL, Web of Science, Scopus and PsycINFO for original articles related to the keywords of 'older persons', 'primary care' and 'inappropriate prescribing'. Our search strategy for PubMed is shown in box 1. Similar search strategies will be used for the other databases. Additionally, we will also hand search the references of review articles related to the topic to retrieve relevant articles which are not captured through our search of the electronic databases.

\section{Study selection}

All potential articles will be retrieved and organised in a data management software (Endnote software, Thomson Reuters). After removing duplicate records, two reviewers will independently screen through the titles and abstracts to retain eligible articles. The first 100 titles and abstracts will be subjected to a calibration exercise between the two reviewers to ensure mutual agreement.

After completing the screening phase, articles that are deemed as relevant by at least one of the reviewers will be subjected to full text review. The two reviewers will 
Box 1 Search strategy for PubMed (MeSH, Medical Subject Headings)

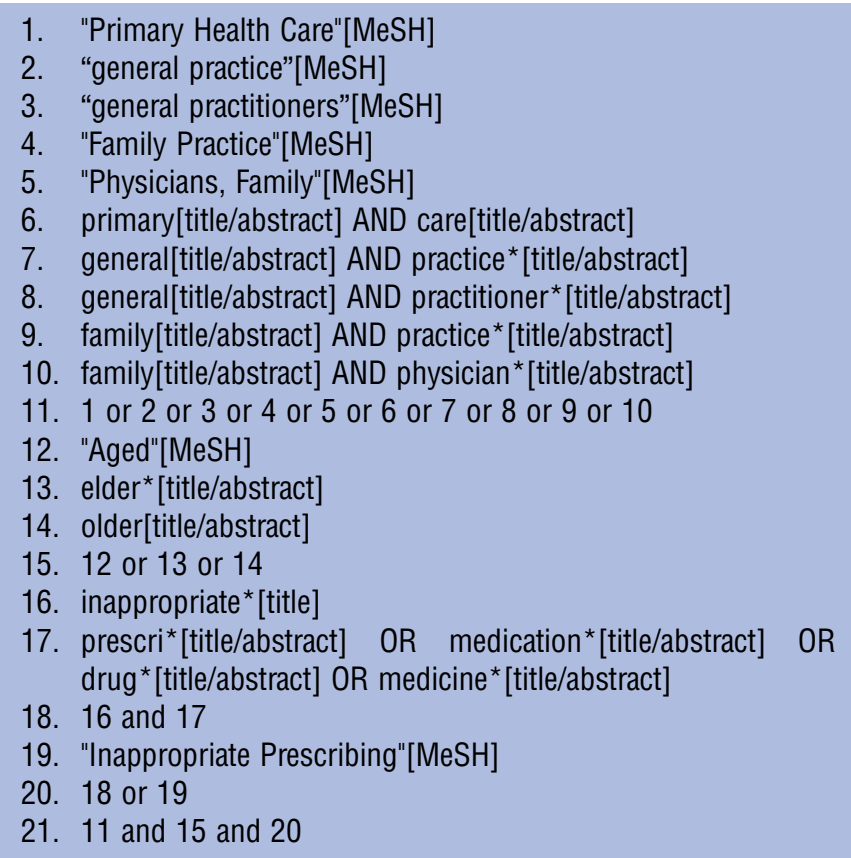

independently confirm the eligibility of these articles based on the full texts. The first $10 \%$ of these full texts will again undergo a calibration exercise by the two reviewers. After the full text review, the included articles will be used for qualitative synthesis. The chancecorrected agreement between the two reviewers will be assessed using Cohen's $\kappa$.

At any point during study selection the reasons for excluding specific articles will be recorded. Moreover, any disagreements between the two reviewers will be resolved by discussion with a third reviewer. If more than one article reported results from the same study, then only the most recent and complete article will be included in the systematic review.

\section{Data extraction}

Data from the selected studies will be extracted by two reviewers independently, and disagreements between the reviewers will be resolved by discussion with a third reviewer. The extracted data will include the following information:

1. Study identification (first author, year of publication, geographical location).

2. Study characteristics (study setting, study design, inclusion criteria, sampling method, sample size, data source of medication prescriptions, study duration, non-response or drop-out rate).

3. Participant characteristics (age, gender, ethnicity, number of comorbidities, number of prescribed medications).

4. Characteristics of inappropriate prescribing (prevalence, working definition, risk factors and adverse outcomes).

\section{Assessment of risk of bias}

The risk of bias for each study will be assessed independently by two reviewers using the Newcastle-Ottawa Scale (NOS) ${ }^{21}$ For case-control and cohort studies, the original 8-item NOS will be used to assess the three key areas of potential bias-selection of participants, comparability of groups and measurement of information. The exact items in the NOS are shown in online supplementary appendices 1 and 2. For cross-sectional studies, only the relevant items in the NOS will be used (see online supplementary appendices 3 and 4). Any disagreements between the two reviewers will be resolved by discussion with a third reviewer.

\section{Statistical analysis}

When two or more studies are included, we will conduct meta-analyses to quantitatively synthesise the results. If there are at least five included studies, we will use the random effects model (DerSimonian and Laird method $)^{22}$ to pool the results because this model does not assume homogeneity among the pooled studies. If there are fewer than five included studies, the random effects model is imprecise in its estimations ${ }^{2324}$ and we will choose the fixed effect model (Mantel-Haenszel method $)^{25}$ instead. When studies report more than one model of statistical adjustment, we will only include the result with the largest number of confounders adjusted for in the statistical model. For each risk factor or adverse outcome of inappropriate prescribing, we will pool the effect size in the form of OR.

We will use the $\mathrm{I}^{2}$ statistic and the $\mathrm{Q}$ test to assess heterogeneity in the pooled studies. In the presence of substantial heterogeneity $\left(\mathrm{I}^{2}>50 \%\right),{ }^{26}$ we will further evaluate the source of heterogeneity using meta-regression analysis and subgroup analyses provided there are at least 10 included studies. The meta-regression and subgroup analyses will be based on the following covariates: year of publication, geographical location, study setting, study design, inclusion criteria, sampling method, sample size, data source of medication prescriptions, study duration, non-response or drop-out rate, age, gender, ethnicity, number of comorbidities, number of prescribed medications, working definition of inappropriate prescribing and study quality.

Separately, we will also evaluate for possible publication bias using funnel plots and Harbord's test ${ }^{27} 28$ if there are at least 10 included studies. In the presence of possible publication bias, we will conduct a sensitivity analysis using the trim and fill method.

All analyses will be done in STATA statistical software package V.13 (Statacorp, 2013) using the commands metaprop (to pool results related to prevalence), metan (to pool results related to risk factors or adverse outcomes), metareg (to conduct meta-regression), metafunnel (to produce funnel plot), metabias (for Harbord's test) and metatrim (for the trim and fill method).

\section{Assessment of quality of evidence}

We will use the Grading of Recommendations Assessment, Development and Evaluation (GRADE) approach to 
report the quality of evidence on the risk factors and adverse outcomes of inappropriate prescribing. Based on five key domains (methodology quality, directness of evidence, heterogeneity, precision of effect estimates and risk of publication bias), we will classify the quality of evidence into one of four levels-high, moderate, low and very low. ${ }^{29}$

\section{ETHICS AND DISSEMINATION}

This systematic review will provide the consolidated evidence to support policymakers to redesign the processes in primary care and enhance medication safety for older persons, thus reducing the preventable and iatrogenic risk to older persons in primary care.

The review will be reported in accordance with the recommendations of the PRISMA statement. ${ }^{19}{ }^{20}$ The results will be disseminated through conference presentations and publications in peer-reviewed journals.

Contributors TML and CSL conceived the idea for this systematic review. TML developed the methodology and search strategy. TML and CSL wrote the manuscript and approved the final version of the manuscript.

Funding TML was supported by a research fellowship under the Singapore Ministry of Health's National Medical Research Council (grant number: NMRC/ Fellowship/0030/2016). The funding source had no involvement in any part of the project.

Competing interests None declared.

Provenance and peer review Not commissioned; externally peer reviewed.

Open Access This is an Open Access article distributed in accordance with the Creative Commons Attribution Non Commercial (CC BY-NC 4.0) license, which permits others to distribute, remix, adapt, build upon this work noncommercially, and license their derivative works on different terms, provided the original work is properly cited and the use is non-commercial. See: http:// creativecommons.org/licenses/by-nc/4.0/

\section{REFERENCES}

1. Hyttinen $\mathrm{V}$, Jyrkka J, Valtonen $\mathrm{H}$. A systematic review of the impact of potentially inappropriate medication on health care utilization and costs among older adults. Med Care 2016;54:950-64.

2. O'Connor MN, Gallagher P, O'Mahony D. Inappropriate prescribing: criteria, detection and prevention. Drugs Aging 2012;29:437-52.

3. Kaufmann CP, Tremp R, Hersberger KE, et al. Inappropriate prescribing: a systematic overview of published assessment tools. Eur J Clin Pharmacol 2014;70:1-11.

4. Spinewine A, Schmader KE, Barber N, et al. Appropriate prescribing in elderly people: how well can it be measured and optimised? Lancet 2007;370:173-84.

5. Grober ED, Bohnen JM. Defining medical error. Can J Surg 2005;48:39-44.

6. Sweeney J, Laoire FO, Linehan G, et al. A review of potentially inappropriate prescribing in over 65's in livinghealth clinic. Ir Med J 2015;108:180-1.

7. Moriarty F, Bennett K, Fahey T, et al. Longitudinal prevalence of potentially inappropriate medicines and potential prescribing omissions in a cohort of community-dwelling older people. Eur J Clin Pharmacol 2015;71:473-82.

8. Bregnhoj L, Thirstrup S, Kristensen MB, et al. Prevalence of inappropriate prescribing in primary care. Pharm World Sci 2007;29:109-15.

9. Dalleur O, Boland B, De Groot A, et al. Detection of potentially inappropriate prescribing in the very old: cross-sectional analysis of the data from the BELFRAIL observational cohort study. BMC Geriatr 2015;15:156.

10. Bradley MC, Motterlini N, Padmanabhan S, et al. Potentially inappropriate prescribing among older people in the United Kingdom. BMC Geriatr 2014;14:72.

11. Perri M III, Menon AM, Deshpande AD, et al. Adverse outcomes associated with inappropriate drug use in nursing homes. Ann Pharmacother 2005;39:405-11.

12. Golden AG, Preston RA, Barnett SD, et al. Inappropriate medication prescribing in homebound older adults. J Am Geriatrics Soc 1999;47:948-53.

13. Aparasu RR, Mort JR. Inappropriate prescribing for the elderly: beers criteria-based review. Ann Pharmacother 2000;34:338-46.

14. Hamilton HJ, Gallagher PF, O'Mahony D. Inappropriate prescribing and adverse drug events in older people. BMC Geriatr 2009;9:5.

15. Lau DT, Kasper JD, Potter DE, et al. Hospitalization and death associated with potentially inappropriate medication prescriptions among elderly nursing home residents. Arch Intern Med 2005;165:68-74.

16. Makary MA, Daniel M. Medical error-the third leading cause of death in the US. BMJ 2016;353:i2139.

17. Santos AP, da Silva DT, dos Santos Junior GA, et al. Evaluation of the heterogeneity of studies estimating the association between risk factors and the use of potentially inappropriate drug therapy for the elderly: a systematic review with meta-analysis. Eur J Clin Pharmacol 2015;71:1037-50.

18. Tommelein E, Mehuys E, Petrovic M, et al. Potentially inappropriate prescribing in community-dwelling older people across Europe: a systematic literature review. Eur J Clin Pharmacol 2015;71:1415-27.

19. Moher D, Shamseer L, Clarke M, et al. Preferred reporting items for systematic review and meta-analysis protocols (PRISMA-P) 2015 statement. Sys Rev 2015;4:1.

20. Shamseer L, Moher D, Clarke M, et al. Preferred reporting items for systematic review and meta-analysis protocols (PRISMA-P) 2015: elaboration and explanation. BMJ 2015;349:g7647.

21. Wells GA, Shea B, O'Connell D, et al. The Newcastle-Ottawa Scale (NOS) for assessing the quality of nonrandomised studies in meta-analyses. http://www.ohri.ca/programs/clinical_epidemiology/ oxford.htm (accessed 6 Oct 2016).

22. DerSimonian R, Laird N. Meta-analysis in clinical trials. Control Clin Trials 1986;7:177-88.

23. Higgins JP, Thompson SG, Spiegelhalter DJ. A re-evaluation of random-effects meta-analysis. J $R$ Stat Soc Ser A Stat Soc 2009;172:137-59.

24. Guyatt G. Users' guides to the medical literature: a manual for evidence-based clinical practice. 3rd edn. McGraw-Hill Education, 2014.

25. Mantel N, Haenszel W. Statistical aspects of the analysis of data from retrospective studies of disease. J National Cancer Inst 1959;22:719-48.

26. Higgins JP, Thompson SG, Deeks JJ, et al. Measuring inconsistency in meta-analyses. BMJ 2003;327:557-60.

27. Harbord RM, Egger M, Sterne JA. A modified test for small-study effects in meta-analyses of controlled trials with binary endpoints. Stat Med 2006;25:3443-57.

28. Higgins JPT. Cochrane handbook for systematic reviews of interventions. Chichester, UK: Wiley, 2008.

29. Schunemann H, Brozek J, Guyatt G, et al. GRADE handbook for grading quality of evidence and strength of recommendation 2013. http://gdt.guidelinedevelopment.org/app/ (accessed 22 Nov 2016). 